\title{
Estudo de viabilidade técnica financeira de sistema de ar condicionado VRF operante com gás natural
}

\begin{abstract}
Renan Morello Cazotti
Graduação em Engenharia Mecânica pelas Faculdades Integradas Espírito Santense FAESA Rua São Jorge, 355. Alto Lage. Cariacica/ES. CEP: 29.150-525

E-mail: renan.cazotti@gmail.com
\end{abstract}

\begin{abstract}
Pablo Altoé Amorim Mestrado em Engenharia Mecânica pela Universidade Federal do Espírito Santo UFES Professor das Faculdades Integradas Espírito Santense - FAESA Rua São Jorge, 355. Alto Lage. Cariacica/ES. CEP: 29.150-525 E-mail: pablo.jafeng@terra.com.br
\end{abstract}

\section{RESUMO}

Com o atual panorama da matriz energética do país, a busca por novas fontes de energia capazes de suprir as necessidades de setores que operam com eletricidade se apresentam como uma oportunidade de utilização de sistemas com maior viabilidade técnica, concorrendo com aplicações convencionais. O sistema GHP (do inglês: gasheatpump) é uma tecnologia que possui como característica fundamental a utilização de gás natural como fonte de energia. O GHP possui aspectos construtivos semelhantes ao VRV (volume de refrigerante variável) que utiliza eletricidade como fonte de energia (EHP do inglês: eletricheatpump), e se aprimorando quando verificado a diminuição do custo operacional, emissão de $\mathrm{CO}_{2}$ reduzida e consumo elétrico. $\mathrm{O}$ estudo de caso aplicado para uma análise de viabilidade técnica e financeira, através das ferramentas VPL e CAE, é realizado em uma instalação real de VRV de uma empresa privada. Através dos dados obtidos a partir de uma abordagem técnica, o sistema GHP comprova sua eficiência e se apresenta como eficiente solução na aplicação de novos projetos e, em alguns casos, obras de melhoria (retrofit).

Palavras-Chave: Ar Condicionado. VPL. GHP.

Technical financial feasibility study of fair conditioning system with natural gas operant

\section{ABSTRACT}

In the current scenario of Brazil's Energy Matrix, the search for new energy sources able to supply the needs of sectors that operate with electricity, presents an opportunity to 
Estudo de viabilidade técnica financeira de sistema de ar condicionado VRF operante com gás

natural

Renan Morello Cazotti, Pablo Altoé Amorim

use systems with greater technical feasibility, competing with conventional applications. The GHP (gas heat pump) system is a technology that has as its fundamental characteristic the use of natural gas as na energy source. The GHP has similar constructive aspects to the VRF (variable refrigerant flow) that uses electricity as an energy source (EHP - electric heat pump), and improves when it is verified the reduction of operational cost, reduced $\mathrm{CO} 2$ emission and electric consumption. The case study applied to a technical and financial feasibility analysis, through the NPV (net present value) and EAC (equivalent annual cost) tools, is performed in a real VRF installation of a private company. Through the data obtained from a technical approach, it is possible to prove that the GHP system is an efficient solution in the application of new projects and, in some cases, improvement projects (retrofit).

Keywords: Air Conditioning. NPV. GHP.

\section{INTRODUÇÃO}

O Brasil sofreu com uma grave crise de escassez de chuvas no ano de 2015 , com isso os reservatórios das principais hidrelétricas chegaram aos seus piores níveis em décadas. Com este cenário, o governo viu-se obrigado a operar com as termoelétricas, onde seus custos de operação e distribuição são mais elevados quando comparados com as hidrelétricas, gerando assim, um custo maior de energia para o consumidor. Para custear esse aumento, o governo começou a empregar o sistema de bandeiras tarifarias que é explicitada na conta de luz do consumidor. Nesta conjuntura, faz-se necessário analisar fontes de energia alternativas que complementem a produção de energia elétrica, evitando a dependência de hidrelétricas e termoelétricas (Ambiente Energia, 2015).

Como uma solução para a geração de energia, o gás natural aparece como uma fonte econômica, versátil e segura. A melhor solução, portanto, combina menor preço e menor impacto.

No setor energético, o gás natural tem variado suas aplicações, explorando cada vez mais o seu potencial. A geração, cogeração e climatização, são exemplos de como o gás natural pode ser amplamente aproveitado. A partir da queima do gás natural é possível gerar energia elétrica e, com a recuperação dos gases de exaustão, gerar calor ou frio. Neste último caso, a cogeração atenderá ao sistema de ar condicionado 
Estudo de viabilidade técnica financeira de sistema de ar condicionado VRF operante com gás

natural

Renan Morello Cazotti, Pablo Altoé Amorim

por ciclo de absorção, alimentando as unidades internas (Companhia de Gás de São Paulo - COMGÁS, 2015).

O presente estudo de caso tem por objetivo realizar um comparativo entre os sistemas GHP e o EHP, através de análise de Valor Presente Líquido (VPL) e cálculo do Custo Anual Equivalente (CAE) tanto para projetos novos como os de melhorias. Para tarifa de energia elétrica e gás natural, foram adotados dados de mercado da região, para o ano de 2016 e 2017. Os valores adotados e respectivos cálculos são evidenciados neste estudo.

\section{BOMBA DE CALOR A GÁS NATURAL - GHP}

A utilização de sistemas de ar condicionado, movidos a gás natural, podem representar uma redução no consumo de energia elétrica entre 91 e 99\%, além de outras vantagens. Os equipamentos utilizados podem ser divididos em resfriadores de líquido por absorção (Chillers) e bombas de calor do tipo GHP. O primeiro funciona por expansão indireta, ou seja, o gás do processo resfria um meio intermediário (ex: água) que, por sua vez, circula em uma serpentina que irá resfriar o ar a ser insuflado no ambiente. Já o segundo funciona tanto por expansão direta quanto indireta. $\mathrm{Na}$ expansão direta, o gás do processo absorve o calor diretamente do ar do ambiente a ser condicionado (COMGÁS, 2016).

O GHP surgiu no mercado de climatização no Japão, como uma alternativa de redução do consumo de energia elétrica, devido ao déficit de oferta no mercado na época. A diferença básica entre a bomba de calor elétrica e a bomba de calor gás está na força que faz o compressor entrar em funcionamento. As bombas de calor elétricas utilizam um motor elétrico para dar partida no compressor, enquanto as bombas de calor a gás utilizam um motor endotérmico a gás natural para dar partida no compressor (Rajkovic, \& Sentié, 2013).

Segundo Camargo (2014), como o ar condicionado representa uma média de 40 a $60 \%$ do consumo de energia de uma edificação, toda a infraestrutura elétrica com a utilização do GHP é reduzida. Sendo assim, subestações, transformadores, cabos e 
Estudo de viabilidade técnica financeira de sistema de ar condicionado VRF operante com gás

natural

Renan Morello Cazotti, Pablo Altoé Amorim

alimentações sofrem redução, logo, as instalações de gás natural possuem valores reduzidos quando comparados com instalações elétricas.

Recentemente, o sistema de bomba de calor acionada pelo motor a gás (GHP) tornou-se uma opção econômica e um sistema de controle de clima mais atraente do que o ar condicionado convencional, devido à sua vantagem na redução do consumo de combustíveis fósseis e a baixa poluição ambiental em países europeus e asiáticos. O GHP geralmente usa o trabalho produzido pelo motor para acionar uma bomba de calor de compressão a vapor (Mahderekal, Shen, Edward, \& Vineyard, 2012).

Uma maneira óbvia para melhorar a utilização de combustível global é localizar o processo de conversão de combustível mais próximo, onde o calor de exaustão pode ser reaproveitado. Uma vantagem adicional, é que o calor residual do motor de combustão pode ser utilizado no local para fornecer aquecimento suplementar ao ambiente ou aquecimento de água. Portanto, o GHP terá uma alta eficiência de utilização de combustível (Baik, Huang, Lian, Park, \& Yao, 2005).

O GHP é um sistema que poderá satisfazer a demanda do sistema de ar condicionado para médios e grandes edifícios, reduzindo a demanda de energia elétrica de ponta, proporcionando economia de energia em geral. O GHP pode ser um sistema de controle do clima mais atraente do que as bombas de calor convencionais por inúmeras razões citadas abaixo (Mahderekal et al., 2012):

- Operação em velocidade variável: normalmente, o GHP possui ciclo com velocidade modular entre uma velocidade mínima e máxima, para coincidir com a carga requerida. As velocidades mínimas e máximas são decididas pelo desempenho do motor e do compressor. Como resultado, a eficiência de carga parcial de um sistema será alta. As suas perdas sazonais de custo e de ciclos operacionais serão mais baixas do que aqueles de um único sistema de velocidade, comparada com um sistema de controle on-off.

- Recuperação de calor do motor: a eficiência do calor do motor não é muito elevada (cerca de $30 \%$ para motores a gás, atualmente). O calor gerado da combustão é desperdiçado através de gases de escape, refrigeração e do bloco do motor. No entanto, a eficiência do sistema GHP irá ser aumentada pela 
Estudo de viabilidade técnica financeira de sistema de ar condicionado VRF operante com gás

natural

Renan Morello Cazotti, Pablo Altoé Amorim

recuperação deste calor a partir da água de arrefecimento e dos gases de escape.

- Combustível gás natural: GHP também difere de bombas de calor elétricas (EHP) na energia que utilizam, principalmente gás natural ou propano, ao invés de eletricidade. Assim, é preferido um sistema GHP em uma região onde os custos elétricos são altos e gás natural é prontamente disponível.

O GHP, tipicamente, usa o trabalho produzido pelo motor para acionar uma bomba de calor de compressão a vapor. Ao mesmo tempo, o calor rejeitado pelo motor é usado para fins de aquecimento. Assim, o GHP é inerentemente mais eficiente do que o aquecimento de sistemas convencionais atualmente comercializados (por exemplo, bomba de calor elétrica). Uma vez que a alta eficiência energética dos GHP opera com baixo consumo de combustível fóssil, a poluição ambiental pode ser reduzida (Mahderekal et al., 2012).

As fontes de energia utilizadas para GHP, como o gás natural, propano ou gás liquefeito de petróleo (GLP), podem ser menos onerosas do que a eletricidade em muitas localidades, de modo que o GHP se torna uma melhor escolha econômica. Além disso, GHP pode desempenhar papéis sociais e econômicos importantes, equilibrando efetivamente a demanda de eletricidade, atenuando os picos de energia elétrica e ajustando a configuração de energia atualmente empregada (Mahderekal et al., 2012).

\section{VIABILIDADE ECONÔMICA}

Uma das etapas mais importantes na elaboração de um projeto é a análise da viabilidade econômica e financeira (Rebelatto, 2004). Definido os equipamentos, é necessário considerar diversos fatores com o objetivo de realizar um estudo preliminar sobre a viabilidade inicial do projeto, esses fatores são: desempenho do equipamento ou sistema, a durabilidade, manutenção, aspectos ergonômicos e, principalmente, o consumo de energia elétrica ou gás.

A análise da viabilidade econômica utilizado neste estudo é realizada através do Método do Valor Presente Líquido (VPL), que, segundo Samanez (2009), consiste em 
Estudo de viabilidade técnica financeira de sistema de ar condicionado VRF operante com gás

calcular o impacto dos eventos futuros associados a uma alternativa de investimento, isto é, medir o valor presente dos fluxos de caixa gerados pelo projeto ao longo de sua vida útil. O VPL pode ser calculando conforme a Eq. (1):

$$
V P L=-I+\sum_{t=1}^{n} \frac{F C_{t}}{\left(1+k^{t}\right)}
$$

Onde $\mathrm{FC}_{\mathrm{t}}$ representa o fluxo de caixa no t-ésimo período, I é o investimento inicial, $\mathrm{K}$ é o custo do capital, e o somatório indica que deve ser realizada a soma da data 1 até $\mathrm{n}$ dos fluxos de caixa descontado ao período inicial.

Como o projeto não gera retorno financeiro, mas mantem o controle da qualidade do ar no ambiente de trabalho, no presente estudo de caso, são avaliados os custos totais dos sistemas ao longo de um determinado período, neste caso, 20 anos, sendo a vida útil de ambos.

Além da análise pelo Método VPL, é utilizado, também, o Método do Custo Anual Equivalente ( $\mathrm{CAE})$, onde, em determinados projetos ou serviços, quando os benefícios e receitas dificilmente podem ser quantificados em termos monetários, os sistemas são avaliados através dos custos (Samanez, 2009).

Segundo Samanez (2009), a fim de identificar a alternativa de menor custo, o CAE rateia, por unidade de tempo, os custos de investimento, de oportunidade e operacionais para cada sistema. O método supõe que as alternativas estudadas serão substituídas por outras idênticas ao término do seu prazo. A Eq (2) relaciona esses indicadores:

$$
C A E=I *\left[\frac{(1+r)^{n} * r}{(1+r)^{n}-1}\right]+\text { Custo Operacional/ano }
$$

Onde I indica o capital inicial de investimento, $r$ a taxa de desconto anual (custo do capital da empresa ao ano) e n é o tempo de duração do projeto ou vida útil. 
Estudo de viabilidade técnica financeira de sistema de ar condicionado VRF operante com gás

natural

Renan Morello Cazotti, Pablo Altoé Amorim

\section{ESTUDO DE CASO - VIABILIDADE TÉCNICA E ECONOMIA DO SISTEMA DE REFRIGERAÇÃO GHP}

O presente estudo de caso foi aplicado a uma empresa do setor de construção civil, que possui sua sede administrativa situada na cidade de Vitória, no Espírito Santo. O prédio administrativo possui uma área refrigerada de $604 \mathrm{~m}^{2}$, sendo dividido em três pavimentos, com 26 setores e uma taxa de ocupação 175 pessoas.

A carga térmica do edifício é de 51 TR (Toneladas de Refrigeração), definida por projeto de engenharia efetuado por empresa competente. Seu sistema é composto por 3 unidades condensadoras de 14 TR, com simultaneidade de $130 \%$, ou seja, o conjunto pode atingir 56 TR e 32 evaporadoras tipo K7, com carga total equivalente à carga térmica do edifício.

No estudo de caso, é realizado um comparativo entre os sistemas GHP e o EHP, através de análise de VPL e CAE, tanto para projetos novos, como de substituição do atual sistema. Para tarifas de energia elétrica e de gás natural, foram adotados valores conforme mercado da região e das instalações em estudo, para o ano de 2016 e primeiro trimestre de 2017.

A fim de levantar dados pertinentes quanto a valores de manutenção e operação do sistema atual, foram realizadas pesquisas nas instalações da empresa com responsáveis técnicos do sistema. Além disso, foram realizadas entrevistas com três possíveis fornecedores da região dos novos sistemas GHP e EHP, com o intuito de obter informações técnicas e de custos para instalações, manutenção e operação dos equipamentos.

As entrevistas foram realizadas de forma semi-padronizada, onde foram listadas as premissas dos projetos, mas a forma de perguntar (a estrutura das perguntas do questionário) e a ordem em que as questões são feitas variam de acordo com as características de cada entrevistado. 
Estudo de viabilidade técnica financeira de sistema de ar condicionado VRF operante com gás

natural

Renan Morello Cazotti, Pablo Altoé Amorim

\section{RESULTADOS E DISCUSSÃO}

Com base nas informações coletadas no estudo e através de cálculos de consumo, em conjunto com pesquisa de mercado, foi elaborada o Quadro 1, onde se pode observar todas as premissas do projeto, com informações detalhadas dos custos envolvidos.

\begin{tabular}{|c|}
\hline PREMISSAS ADOTADAS \\
\hline 1 - Tarifa de Energia Elétrica - EDP ESCELSA \\
\hline 2 - Carga térmica - 51,0 TR. \\
\hline 3 - Horário de Funcionamento - 8 horas por dia. \\
\hline 4 - Taxa de Ocupação Fora da Ponta - 100\%. \\
\hline 5 - Consumo de Energia VRF Elétrico - 0,92 kWh/TRh. \\
\hline 6 - Consumo de Energia VRF a Gás - 0,092 kWh/TRh. \\
\hline 7 - Consumo de Gás Natural - 0,27 m³/h por TR \\
\hline 8 - Custo do $m^{3}$ do gás natural para climatização - $R \$ 0,9108$ \\
\hline 9 - Custo de energia elétrica - $R \$ 0,6755 / \mathrm{kW} / \mathrm{h}$ \\
\hline 10 - Custo de Manutenção do GHP por ano - $\mathrm{R} \$ 698,00$ por TR \\
\hline 11 - Custo de Manutenção do VRF por ano - R\$582,00 por TR \\
\hline 12 - Investimento para aquisição GHP não instalado - R\$ 7.050,00 por TR \\
\hline 13 - Investimento para aquisição VRF não instalado - $\mathrm{R} \$ 4.683,00$ por TR \\
\hline 14 - Investimento para instalação GHP não instalado - $R \$ 3.674,00$ por TR \\
\hline 15 - Investimento para instalação VRF não instalado - R\$ 3.674,00 por TR \\
\hline 16 - Alíquota de IR da empresa - $27 \%$ \\
\hline 17 - Custo de capital da empresa - $15 \%$ \\
\hline 18 - Vida útil (restante) do sistema EHP operante - 10 anos \\
\hline 19 - Vida útil dos sistemas novos - 20 anos \\
\hline
\end{tabular}

Quadro 1. Premissas adotadas

Fonte: Elaborado pelos autores

Os dados colhidos no Quadro 1 se referem ao projeto especifico, podendo variar de acordo com a complexidade, tamanho, localização e tempo de operação para cada empreendimento onde o estudo for aplicado. 
No Quadro 2, são apresentados os valores em reais de CAPEX (Capital Investido) e OPEX (Custos Operacionais por ano) de ambos sistemas, atendendo as premissas do projeto, tanto para projeto novo quanto para projeto de substituição.

Para projeto de substituição, parte do sistema existente (EHP) é utilizado nas novas instalações de GHP. Assim, pode ser observado que o custo de equipamentos novos e instalação do sistema de substituição é menor do que o custo de instalação de um projeto novo completo. 
Estudo de viabilidade técnica financeira de sistema de ar condicionado VRF operante com gás

natural

Renan Morello Cazotti, Pablo Altoé Amorim

\begin{tabular}{|c|c|c|c|c|}
\hline & \multicolumn{2}{|c|}{ PROJETO NOVO } & \multicolumn{2}{|c|}{$\begin{array}{l}\text { PROJETO DE } \\
\text { SUBSTITUIÇÃO }\end{array}$} \\
\hline $\begin{array}{c}\text { EMPREENDIMENTO: PRÉDIO } \\
\text { ADMINISTRATIVO }\end{array}$ & $\begin{array}{l}\text { EHP (VRF } \\
\text { ELÉTRICO) }\end{array}$ & GHP & $\begin{array}{l}\text { EHP (VRF } \\
\text { ELÉTRICO) }\end{array}$ & GHP \\
\hline $\begin{array}{l}\text { CLASSIFICAÇÃO DE CAPACIDADE DO } \\
\text { SISTEMA }\end{array}$ & \multicolumn{4}{|c|}{ EDP ESCELSA } \\
\hline $\begin{array}{l}\text { Capacidade Térmica Total do } \\
\text { Condicionadores (TR) }\end{array}$ & 51 & 51 & 51 & 51 \\
\hline $\begin{array}{c}\text { Carga Térmica Anual Fora de Ponta } \\
\text { (TRh) }\end{array}$ & 117.504 & 117.504 & 117.504 & 117.504 \\
\hline Carga Térmica Anual - Ponta (TRh) & 0 & 0 & 0 & 0 \\
\hline Carga Térmica Anual - (TRh) & 117.504 & 117.504 & 117.504 & 117.504 \\
\hline Consumo de Energia Elétrica (kWh/TRh) & 0,92 & 0,092 & 0,92 & 0,092 \\
\hline \multicolumn{5}{|c|}{ DESPESAS DE CAPITAL (CAPEX) } \\
\hline Investimento Equipamentos AC & $\mathrm{R} \$ 201.369$ & $\mathrm{R} \$ 303.150$ & - & $\mathrm{R} \$ 263.000$ \\
\hline $\begin{array}{l}\text { Investimento para Instalação } \\
\text { Equipamentos AC }\end{array}$ & $R \$ 158.000$ & $R \$ 158.000$ & - & $\mathrm{R} \$ 48.000$ \\
\hline Investimento adicional na parte elétrica & $\mathrm{R} \$ 29.505$ & $\mathrm{R} \$ 18.945$ & - & $\mathrm{R} \$ 18.945$ \\
\hline Custo Total de CAPEX & $\mathbf{R} \$ 388.874$ & $\mathrm{R} \$ 480.095$ & $\mathbf{R} \$ 0$ & $\mathbf{R} \$ 329.945$ \\
\hline DIFERENÇA DE INVESTIMENTO & & $-\mathrm{R} \$ 91.221$ & & $-\mathrm{R} \$ 329.945$ \\
\hline \multicolumn{5}{|c|}{ CUSTOS OPERACIONAIS (OPEX) } \\
\hline \multicolumn{5}{|c|}{ CONSUMO DE ENERGIA ELÉTRICA } \\
\hline Demanda do Sistema (kW) & 47 & 4,7 & 47 & 4,7 \\
\hline Consumo Anual Fora de Ponta (kWh) & 108.104 & 10.810 & 108.104 & 10.810 \\
\hline Consumo Anual Ponta (kWh) & 0 & 0 & 0 & 0 \\
\hline Custo Especifíco EE (R\$/kWh) & 0,6755 & 0,6755 & 0,6755 & 0,6755 \\
\hline Custo Anual Energia Elétrica & $\mathrm{R} \$ 73.024$ & R\$ 7.302 & $\mathbf{R} \$ 73.024$ & $\mathbf{R} \$ 7.302$ \\
\hline \multicolumn{5}{|c|}{ CONSUMO DE GÁS NATURAL REFRIGERAÇÃO } \\
\hline Consumo de Gás Natural (m³/h) & - & 0,27 & - & 0,27 \\
\hline Consumo Anual Sistema AC $\left(\mathrm{m}^{3}\right)$ & - & 31.726 & - & 31.726 \\
\hline Custo Específico $\left(\mathrm{R} \$ / \mathrm{m}^{3}\right)$ & - & $\mathrm{R} \$ 0,9108$ & - & $\mathrm{R} \$ 0,9108$ \\
\hline Custo Fixo Anual & - & $\mathrm{R} \$ 3.633$ & - & $\mathrm{R} \$ 3.633$ \\
\hline Custo Anual Gás Natural & - & $\mathbf{R} \$ 32.529$ & - & $\mathbf{R} \$ 32.529$ \\
\hline \multicolumn{5}{|c|}{ CUSTO DE MANUTENÇÃO } \\
\hline $\begin{array}{l}\text { Custo de Manutenção Anual Sistema } \\
\text { AC }\end{array}$ & $\mathbf{R} \$ 25.000$ & $\mathbf{R} \$ \mathbf{3 0 . 0 0 0}$ & $R \$ 25.000$ & $\mathbf{R} \$ \mathbf{3 0 . 0 0 0}$ \\
\hline Custo OPERACIONAL TOTAL (OPEX) & $R \$ 98.024$ & $\mathbf{R} \$ 69.831$ & $R \$ 98.024$ & $\mathbf{R} \$ 69.831$ \\
\hline ECONOMIA ANUAL & REFERÊNCIA & $\mathrm{R} \$ 28.194$ & REFERÊNCIA & $\mathrm{R} \$ 28.194$ \\
\hline & & $28,76 \%$ & & $28,76 \%$ \\
\hline
\end{tabular}

Quadro 2. Levantamento financeiro entre EHP x GHP

Fonte: Elaborado pelos autores

Conforme dados do Quadro 2 e utilizando as Equações 1 e 2, o Quadro 3 apresenta os resultados para VPL referente aos gastos e CAE, para vida de 20 anos e custo de capital da empresa de $15 \%$. 
Estudo de viabilidade técnica financeira de sistema de ar condicionado VRF operante com gás natural

Renan Morello Cazotti, Pablo Altoé Amorim

Pode-se observar que, quando os sistemas EHP e GHP são avaliados, desde a fase de instalação, como projetos novos, o consumo de energia elétrica do GHP é inferior, e a política da tarifação do gás natural é vantajosa em comparação com a energia elétrica.

\begin{tabular}{|c|cc|cc|}
\hline \multicolumn{5}{|c|}{ PROJETO NOVO } \\
\hline Análise & \multicolumn{2}{|c|}{ EHP } & $-\mathrm{R} \$$ & $917.190,38$ \\
\hline $\mathrm{VPL}$ & $-\mathrm{R} \$$ & $1.002 .438,71$ & $-\mathrm{R} \$$ & $146.531,68$ \\
\hline $\mathrm{CAE}$ & $-\mathrm{R} \$$ & $160.151,08$ & & \\
\hline
\end{tabular}

\section{Quadro 3. Comparativo de VPL e CAE entre EHP x GHP}

Fonte: Elaborado pelos autores

O estudo analisa, também a substituição do sistema existente EHP por um novo sistema GHP através de VPL. Conforme entrevistas e levantamento de campo, foram obtidos os dados do sistema atual, onde a máquina antiga pode operar por mais 10 anos e que seu valor contábil é de aproximadamente $R \$ 80.000,00$, depreciado linearmente em 10 anos. A máquina nova custa $R \$ 263.000,00$ (depreciado ao longo de 20 anos), somando instalações e sistema elétrico, tem um custo total de $R \$ 329.945$. Após 10 anos, a máquina GHP pode ser vendida por $R \$ 131.500,00$. Com esses dados, foi possível calcular o Fluxo de Caixa Livre (FCL), apresentado no Quadro 4.

\begin{tabular}{|l|c|c|c|c|c|c|c|}
\hline \multicolumn{7}{|c|}{ PROJETO DE SUBSTITUIÇÃO } \\
\hline \multicolumn{1}{|c|}{ EHP Novo } & Ano 0 & Ano 1 & Ano 2 & $\ldots$. & Ano 9 & Ano 10 & Liquidação \\
\hline Investimento líquido & $-249.945,00$ & & & & & & $131.500,00$ \\
\hline $\begin{array}{l}\text { + Diminuição dos custos } \\
\text { operacionais }\end{array}$ & & $28.194,00$ & $28.194,00$ & & $28.194,00$ & $28.194,00$ & \\
\hline - Depreciação incremental & & $-5.150,00$ & $-5.150,00$ & $\ldots$ & $-5.150,00$ & $-5.150,00$ & \\
\hline Lucro antes de juros e IR & & $23.044,00$ & $23.044,00$ & $\ldots$. & $23.044,00$ & $23.044,00$ & \\
\hline - Impostos (27\%) & & $-6.221,88$ & $-6.221,88$ & $\ldots$. & $-6.221,88$ & $-6.221,88$ & \\
\hline + Depreciação incremental & & $5.150,00$ & $5.150,00$ & $\ldots$. & $5.150,00$ & $5.150,00$ & \\
\hline Fluxo de Caixa Livre (FCL) & $-2499.45,00$ & $21.972,12$ & $21.972,12$ & $\ldots$. & $21.972,12$ & $21.972,12$ & $131.500,00$ \\
\hline
\end{tabular}

\section{Quadro 4. Fluxo de Caixa Livre (valores apresentados em moeda Real)}

Fonte: Elaborado pelos autores 
Estudo de viabilidade técnica financeira de sistema de ar condicionado VRF operante com gás

natural

Renan Morello Cazotti, Pablo Altoé Amorim

Através da Equação 1, utilizando o FCL de - $R \$ 249.945,00$ e tempo de estudo de 10 anos, foi possível calcular um VPL de - $R \$ 139.672,01$, para substituição do sistema antigo EHP por um novo GHP. Esse valor negativo indica que o projeto não é viável. Essa avaliação deve ser realizada todo ano, devido às mudanças dos valores de CAPEX e OPEX, até que se obtenha um valor do VPL positivo, apontando viabilidade na substituição.

Para ambos projetos, os resultados podem ser ainda melhores, dependendo de algumas condições que são evidenciados a seguir.

Empreendimentos que operam dentro do horário de ponta, no qual a tarifação de energia elétrica é muito mais elevada, a economia proporcionada pelo GHP apresenta resultados melhores do que em projetos sem esta característica, tornando seu OPEX mais atrativo e diminuindo o tempo de retorno do investimento aplicado.

Os meios de recuperação de calor utilizados neste estudo não consideram a recuperação de todo o calor residual do motor. Para melhorar ainda mais a eficiência da fonte energética, poderia ser acrescentada uma bobina de recuperação de calor interior para o modo de aquecimento de outros locais, ou utilizar o calor residual restante para aquecimento de água na utilização de chuveiro, lavanderia em geral, piscinas, entre outras funcionalidades.

Uma alternativa que deve ser levada em consideração é a utilização de um gerador a gás para o sistema EHP em horários de ponta, diminuindo o consumo do sistema pelo fato do gás natural não possuir variação de valores na tarifa em horário de ponta e possuir um custo de combustível menor em comparação com diesel. Com a utilização de um gerador a gás, surge a opção de trabalhar com cogeração, utilizando mais uma fonte energética, tornando a operação mais segura e econômica.

O gerador abastecerá de energia elétrica o EHP durante este intervalo de tempo. Portanto, torna-se necessário realizar outro estudo para analisar a viabilidade da aquisição deste novo componente, já que implica no aumento do custo do CAPEX do sistema. Mas esta alternativa pode ser de grande valia para indústrias, shoppings, hotéis, hospitais, entre outros estabelecimentos que consomem grande quantidade de energia no horário de ponta. 
Estudo de viabilidade técnica financeira de sistema de ar condicionado VRF operante com gás

natural

Renan Morello Cazotti, Pablo Altoé Amorim

\section{CONCLUSÃO}

Pelo fato do GHP utilizar uma energia primária (gás natural) como combustível de operação, seu funcionamento se torna mais rentável, pelo baixo consumo de energia elétrica, constituindo um diferencial, já que sua tecnologia proporciona baixo impacto ao meio ambiente, sua descarga de $\mathrm{CO}_{2}$ na atmosfera é reduzida em até $20 \%$, quando comparado a outros sistemas de refrigeração.

As informações coletadas com fornecedores são de que o sistema GHP possui uma taxa de manutenção mais elevada, porém com maior confiabilidade, gerando maiores garantias de operação, proporcionando maior satisfação ao cliente final.

Os resultados foram alcançados e mensurados com a utilização das ferramentas de análise VPL e CAE, que avaliam os custos de capital e custos operacionais de cada sistema de refrigeração, determinado, de forma simples e objetiva, o período estimado para efetuar retorno financeiro.

Com a análise e o estudo direcionado, foi possível identificar o processo de operação das duas tecnologias, evidenciando que o sistema GHP possui um custo inicial mais elevado em comparação ao EHP. Neste estudo de caso, ficou constatado que o VPL do empreendimento para um projeto de melhoria fica negativo, tornando inviável a substituição do sistema antigo. Em decorrência deste fato, o estudo deve ser realizado novamente no próximo ano, com atualização dos valores de CAPEX e OPEX.

Para análise comparativa de um empreendimento novo, o sistema GHP proporcionou resultados satisfatórios. Isto torna este sistema rentável, quando comparado com o sistema EHP, devido, primeiramente, ao baixo custo do gás natural, aplicado pela concessionária na região, e ao alto custo de energia elétrica existente. Além disso, se pode citar o reaproveitamento do subproduto do motor a gás para aquecimento da água quente que não é aplicada nesse estudo, mas pode ser utilizada em outras obras e a redução da infraestrutura elétrica. 
Estudo de viabilidade técnica financeira de sistema de ar condicionado VRF operante com gás

natural

Renan Morello Cazotti, Pablo Altoé Amorim

\section{REFERÊNCIAS}

Ambiente Energia. (2015). Crise energética e as diferentes visões sobre o problema. Recuperado de: <https://www.ambienteenergia.com.br/index.php/2015/02/criseenergetica-e-diferentes-visoes-sobre-o-problema/25550>

Baik, Y., Huang, W., Lian, Z., Park, S., \& Yao, Y. (2005). Conception of combination of gas-engine-driven heat pump and water-loop heat pump system. International Journal of Refrigeration, Int J Refrigeration, 28, p. 2.

Camargo, R. C. A. (2014). Sistemas de condicionadores de ar baseados em bombas de calor a gás - GHP. Anais do Congresso Internacional de Ar, Refrigeração, Aquecimento e Ventilação- ASBRAV, RS, 9, pp. 4-8.

Companhia de Gás de São Paulo - COMGÁS. (2015). Gás natural: uma alternativa real para o Brasil. Revista Ar Condicionado LG, São Paulo, 5, p. 14.

Mahderekal, I., Shen, B., Edward A., \& Vineyard, E. A. (2012). System Modeling of Gas Engine Driven Heat Pump. International Refrigeration and Air Conditioning Conference at Purdue, July 16-19, pp.1-2.

Rajkovic, D., \& Sentié, M. (2013). Comparison of working (operating) efficiency of electric and gas heat pump regarding to renewable heat source. Taylor \& Francis Group, London, p. 3.

Rebelatto, D. A. do N. (2004). Projeto de investimento: com estudo de caso completo na área de serviços. São Paulo, pp. 141-230.

Samanez, C. P. (2009). Engenharia econômica. São Paulo: Pearson Prentice Hall, pp.36-42 e pp. 100-108.

Data de Submissão: 29/06/2016

Data de Aceite: 20/06/2017 